wurden kürzlich im Rahmen des Heilund Hilfsmittelversorgungsgesetzes untersagt.

\section{MMW-KOMMENTAR}

Die Studie äußert, ohne dies ausdrücklich so zu formulieren, den Verdacht, dass die Aufsichtsbehörden unerlaubte finanzielle Anreize für die Diagnosestellung in Verträgen zur hausarztzentrierten Versorgung (HZV) nach $\S 73 b$ SGB V übersehen hätten. Das Schlupfloch für Diagnosemanipulationen sollen die Chronikerpauschalen in der HZV sein, weil diese nur dann gezahlt werden, wenn eine gesicherte ICD-Kodierung einer der 80 Krankheiten des Morbi-RSA vorliegt.

Grundsätzlich gilt im Gesundheitswesen aber, dass ein Vergütungsanspruch für Leistungen nur über einen Dokumentationsnachweis realisiert werden kann. Schon im EBM wird deshalb für die Behandlung und Betreuung eines Patienten mit mindestens einer lebensverändernden chronischen Erkrankung ein Zuschlag nach Nr. 03220 zur Versichertenpauschale gewährt-aber nur gezahlt, wenn die zu behandelnde chronische Erkrankung auch richtig auf dem Abrechnungsschein des Arztes dokumentiert wurde. Es handelt sich somit um ein gängiges Verfahren. Dass solche Diagnosen unabhängig von der jeweiligen Vertragsform im ambulanten wie auch im stationären Bereich fehlerhaft sein können, ist völlig normal. Sie müssen dann natürlich korrigiert werden. Aber weder die $H Z V$-Verträge noch der EBM intendieren eine falsche Diagnosestellung durch Ärzte. So gesehen braucht niemand das Ergebnis dieser Studie zu fürchten. Wer verantwortungsvoll seine Leistungen kodiert, braucht nichts zu befürchten - auch dann nicht, wenn diese Sorgfalt mit einer besseren Honorierung verbunden ist.

\title{
So gesehen lohnt sich die Kleinchirurgie
}

Die allgemeinen diagnostischen und therapeutischen Leistungen des EBMAbschnitts II.2 unterliegen sämtlich dem Regelleistungsvolumen (RLV) oder den qualifikationsgebundenen Zusatzvolumina (QZV) - und damit einer Mengenbegrenzung. Wird ein QZV nicht ausgefüllt, werden die verbleibenden Anteile dem RLV zugeschlagen.

Dort allerdings gehen sie verloren, wenn das vorhandene Honorarvolumen nicht abgerufen wird. Das kann leicht passieren, da im hausärztlichen Bereich zur Füllung des RLV nur wenige Pauschalen und die Gesprächsleistung nach Nr. 03230 zur Verfügung stehen. Das spricht also dafür, auch auf allgemeine Leistungen zu setzen.
Das Nachrechnen lohnt sich auch unter dem Aspekt der Zeitvorgaben, die momentan von den KVen verschärft überprüft werden. Das Gespräch nach Nr. 03230 schlägt nämlich mit einer hohen Zeitvorgabe zu Buche, obgleich es im Rahmen eines eigenen Budgets vergütet wird - mitunter also gratis erbracht wird. Bei den allgemeinen Leistungen ist das anders.

\section{MMW-KOMMENTAR}

Ein gutes Beispiel ist die Kleinchirurgie. So wird der kleinchirurgische Eingriff I nach Nr. 02300 mit 6 Euro vergütet, hat aber nur eine Plausibilitätszeit von 3 Minuten. Selbst beim kleinchirurgischen Eingriff II nach Nr. 02301 sind es nur 6 Minuten, das Honorar liegt aber schon bei 13,58 Euro - und damit deutlich über den 9,48 Euro bei der Gesprächsleistung nach Nr. 03 230. Erst der kleinchirurgische Eingriff III nach Nr.03202 kommt auf dieselbe Plausibilitätszeit von 10 Minuten wie die Gesprächsziffer, wird aber bereits mit 25,17 Euro vergütet.

So gesehen lohnt es sich aufjeden Fall, solche Leistungen, wenn sie anfallen, auch selbst zu erbringen - und nicht aus falscher Angst vor einer Leistungserbringung zum Nulttarif an spezialisierte Ärzte zu überweisen. Dabei sollte man auch nicht vergessen, dass selbst bei einer Überschreitung des RLV bzw. des QZV die entsprechenden Leistungen, wenn auch zu einem niedrigeren Punktwert, weiter vergütet werden, ohne dass der Vorteil bei den Zeitvorgaben wegfällt.

\section{Tab. 1 Auch die Plausi-Zeit fällt bei der Bewertung einer Leistung wie der Kleinchirurgie ins Gewicht}

\begin{tabular}{|l|l|l|l|l|}
\hline EBM & Legende & Euro & Minuten \\
\hline 03230 & $\begin{array}{l}\text { Problemorientiertes ärztliches } \\
\text { Gespräch }\end{array}$ & 9,48 & 10 \\
\hline 02300 & Kleinchirurgischer Eingriff I & 6,00 & 3 \\
\hline 02301 & Kleinchirurgischer Eingriff II & 13,58 & 6 \\
\hline 02302 & Kleinchirurgischer Eingriff III & 25,17 & 10 \\
\hline
\end{tabular}

